\title{
Laguerre-Gaussian beams shaping by binary phase plates as illumination sources in micro-optics
}

\author{
Francisco Javier Salgado-Remacha \\ Universidad de Zaragoza \\ Departamento de Física Aplicada, Facultad de Ciencias, \\ C/ Pedro Cerbuna 12, 50009 Zaragoza (Spain) \\ E-mail: fjsalgado@unizar.es
}

The lack of intensity in the central region of Laguerre-Gaussian beams reduces its applicability as illumination sources. For this reason, it is usual to shape a LaguerreGaussian beam to a nearly-Gaussian beam using a binary phase mask. The behaviour of this rectified Laguerre-Gaussian beam is analysed in this work in the Fresnel regime. A comparison between diverse Laguerre-Gaussian beams with rectified Laguerre Gaussian beams shows that there appear two differentiate regions along the propagation axis: first transition region (for lower distances) with a flat intensity distribution, and a second region (for longer distances) where the rectified Laguerre-Gaussian tends to a Gaussian shape. The results of this work are very valuable for the use of this kind of beams in micro-optical applications.

Keywords: (140.3300) Laser beam shaping; (070.7345) Wave propagation; (070.2580); Paraxial wave optics; (250.7260) Vertical cavity surface emitting lasers 


\section{Introduction}

In the recent years, several efforts have been made in order to miniaturize optical components and systems. In this sense, vertical-cavity surface-emitting lasers (VCSELs) [1] represent a good choice as illumination source, due the small size and its mechanical properties. For this reason, VCSELs have been extensively used in several micro opto-electronical applications [2]-[5]. However, when relatively high output powers are required, VCSELs (and other laser resonators) emit usually in a high-order Gaussian beam [6]-[8]. Under cylindrical symmetry, these kinds of beams are referred as Laguerre-Gaussian beams, $L G_{p}^{m}$, where $p$ is the radial order and $m$ is the azimuthal order [9]. The fundamental mode $L G_{0}^{0}$ corresponds to a pure Gaussian beam. Higher orders $L G_{p}^{m}$ (specifically $L G_{0}^{m}$ beams) can present some kind of singularity in the phase at the central position, and consequently the beam intensity vanishes at the central region [9]. This fact reduces the applications of this kind of beams in fields such as communications, sensing or micro optics. For example, Fresnel-type Computer Generated Holograms $(\mathrm{CGH})$ deflect an illumination beam producing an intensity pattern in the Fresnel regime [10]. The illumination with a non-fundamental $L G_{p}^{m}$ with no intensity in the central region reduces drastically the efficiency of the system.

Several solutions have been proposed to transform a $L G_{p}^{m}$ into a fundamental Gaussian beam, [11]-[22], regardless the nature of the illumination source. The simplest transformation is made with a binary phase plate having alternating 0 and $\pi$ phase shifts, eliminating the singularity of the phase. This solution is also the best solution for micro-optical application, minimizing the size of the system. Thus, a Gaussian-like beam is obtained in the far field, or when the beam is focused. In general, the published works are centered on the focalization capabilities of the transformed beams and its spatial characteristics in the far field. However, 
it is not clear at which point along the propagation axis a corrected beam can be considered as a near-Gaussian beam with stable intensity at the central region. For a considerable number of micro optical applications, the common feature of the systems implies light travel lengths in the range of Fresnel approach. In this sense, unlike previous works, we analyze in this work the propagation under Fresnel approach, in order to obtain a good description of the propagation of a rectified $L G_{p}^{m}$ beam.

For the numerical analysis, we have used the "Beam Propagation Method" (BPM) [23]. It consists of a split-step numerical method for the simulation of light propagation under Fresnel approach. Thus, we consider scalar and paraxial domain, and we will assume in the following that laser beams have transversal dimensions small enough to consider them as paraxial beams and the angular spectrum of the amplitude distribution is located around the axis of propagation). Moreover, we will assume that the amplitudes of the beams are scalar quantities, and we will not consider polarization effects. These assumptions are in concordance with the common features of VCSELs and micro opto-electronical devices, and do not reduce the validity of our work.

This paper is organized as follows: first we explain in Section 2 the characteristics of the Laguerre-Gaussian beams. Next, in Section 3 we analyze the behavior of the rectified Laguerre-Gaussian beams under Fresnel approach. Finally, in Section 4 we summarize the main conclusions that can be extracted from this work.

\section{Theoretical fundamentals}

A Laguerre-Gaussian beam $L G_{p}^{m}$ of orders $p$ and $m$ (with beam waist radius $\omega_{0}$ ) in cylindrical coordinates $(z, r, \theta)$ has its electric field amplitude at $z=0$ defined by [24] 


$$
E(z=0, r, \theta)_{z=0}=\left(\sqrt{2} \frac{r}{\omega_{0}}\right)^{m} L_{p}^{m}\left(\frac{2 r^{2}}{\omega_{0}^{2}}\right) \exp \left(-\frac{r^{2}}{\omega_{0}^{2}}\right) \exp (-i m \theta)
$$

where $L_{p}^{m}$ is the Laguerre polynomial with mode orders $p$ and $m$ [25]. When $p=m=0$ the expression refers to a pure Gaussian beam. The beam waist width $\omega_{0}$ coincides with the Gaussian width of the beam only for the fundamental mode. For higher orders, its width can be calculated by means of by [26]

$$
\omega_{p m}=\omega_{0} \sqrt{2 p+m+1}
$$

Another important parameter related to Gaussian beams is the Rayleigh Range. For the fundamental mode, and being $\lambda$ the wavelength, this parameter is expressed as

$$
z_{R}=\frac{\pi \omega_{0}^{2}}{\lambda}
$$

which gives a description of the behavior of the beam along the propagation distance. The width of a fundamental Gaussian beam at $z_{R}$ is $w\left(z=z_{R}\right)=\sqrt{2} w_{0}$. For example, for a Gaussian beam with $\omega_{0}=5 \mu \mathrm{m}$ and $\lambda=0.6 \mu \mathrm{m}$, eq. (3) results $z_{R}=130 \mu \mathrm{m}$. We will use the Rayleigh Range in order to generalize our analysis along the distance propagation.

Some examples of $L G_{p}^{m}$ beams at $z=0$ are collected in Figure 1, where several intensity and phase patterns for $L G_{p}^{m}$ beams with different values of $p$ and $m$ are drawn. The intensity patterns have been obtained as the square of the complex amplitude field distribution. As it is shown, for $p=m=0$ we obtain a pure Gaussian beam with a constant value of the phase. On the contrary, when the value of $p$ increases, corresponding concentric rings appear in the phase distribution. A profile along the radial coordinate from the central point to an extreme will change its value $p$ times between $-\pi / 2$ and $\pi / 2$. The intensity pattern has the form of a central peak surrounded by secondary concentric rings if $m=0$. On the contrary, when the 
value of $m$ increases, there is not radial dependence in the phase distribution, but a profile along the azymuthal coordinate will change its value $m$ times between $-\pi / 2$ and $\pi / 2$ when $2 \pi$ radians are covered. Thus, a singularity appears at the central position, which is the origin of the lack of intensity in the central region. When not $p$ nor $m$ vanish, we can appreciate both effects.

When a $L G_{p}^{m}$ beam propagates, the singularity in phase remains along the propagation. As example, and considering only the paraxial domain, some simulations by means of BPM are here carried out. We introduce as illumination field one of the modes shown in Figure 1. Then, we propagate along a certain distance $z$ from the origin. Finally, we take the profiles along $x$-axis at $y=0$ for each distance, and we plot together these profiles. In Figure 2 are drawn a propagation for a $L G_{0}^{0}$ beam and for $L G_{0}^{1}$ beam. As we have commented, the singularity when $m \neq 0$ remains along the propagation, and consequently these kind of beams are not convenient as illumination sources. In the next section we will show a method to remove this singularity, and an analysis of the rectified beam.

\section{Rectified Laguerre-Gaussian beams}

The procedure used in this work to correct the singularity is quite simple: we introduce a binary phase mask at $z=0$ in the form of the complementary image of the beam phase pattern [22]. In other words, wherever the beam phase has a value of $-\pi / 2$, the correction mask has a value of $\pi / 2$ and vice versa. Thus, the corrected beam (also called "rectified" Laguerre-Gaussian beam, referred here as $R L G_{p}^{m}$ ) has the same intensity pattern, but with a constant phase pattern. Binary phase mask manufacturing is a reliable and attainable technology, and advantageous in terms of cost-efficiency. 
Figure 3 shows the propagation of a $R L G_{0}^{1}$ beam and a $R L G_{0}^{4}$ beam. As can be seen, there is a redistribution of the intensity as the beam propagates. Thus, we see that the intensity at the central point grows with the distance of propagation for both cases. At the same time, the lobes-structure is lost with the propagation. The profiles along $x$-and $z$-axis at $y=0$ are also plot in Figure 4.

It is remarkable that this kind of beams do not correspond to a pure Gaussian beam, rather to generalized Gaussian beam. Thus, eq. (2) is no longer valid for the description of the beam width. In order to obtain a proper description of the $R L G_{p}^{m}$ beams, we can make use of a generalized width, based on second-order moments of the irradiance patterns [26]. Let us assume an amplitude distribution along one dimension, $E(x)$. We define first the "center of mass" of this distribution as

$$
x(E)=\frac{\int_{-\infty}^{\infty}|E(x)|^{2} x d x}{\int_{-\infty}^{\infty}|E(x)|^{2} d x}
$$

Then, the width of the $R L G_{p}^{m}$ beam is defined as

$$
\omega_{x}(E)=2 \sqrt{\frac{\int_{-\infty}^{\infty}|E(x)|^{2}[x-x(E)]^{2} d x}{\int_{-\infty}^{\infty}|E(x)|^{2} d x}},
$$

and similarly for $\omega_{y}(E)$ along the $y$-axis. We make use of $\omega_{x}(E)$ rather than $\omega_{y}(E)$ since we always have lobes along the $x$-axis for any $L G_{p}^{m}$ mode with $m \neq 0$. Using this expression, we have obtained the Gaussian width of several $R L G_{0}^{m}$ beams with different $m$ orders (see Figure 5a). As can be shown, for the fundamental mode (where no correction has been made, since the phase pattern has no singularity) corresponds to the behavior of a 
fundamental Gaussian beam. Also the $R L G_{0}^{1}$ beam shows a similar behavior, but with higher width and divergence as the fundamental mode. It should be noted that the $L G_{0}^{1}$ phase pattern has not dependence along the $y$-direction, and is a special case. On the contrary, for rectified beams of higher orders, two regions can be differentiated: first, there is a transition region between a $L G_{0}^{m}$ mode and a near-fundamental Gaussian beam. In this region $\omega_{x}(E)$ has a maximum and a minimum. This first region is shorter when $m$ increases, but the width is higher when $m$ increases. In the second region, the width follows a dependence similar to a Gaussian beam. In the case of $R L G_{0}^{2}$, the beam even shows a lower divergence as the fundamental Gaussian beam. Higher values of $m$ the divergence of the beams decreases with $m$. For any case, the transition between both regions are located at a distance lower than $2 z_{R}$. A study of the characteristics of $R L G_{p}^{m}$ beams in the far field can be founded in [22]. Also, Figure 5b shows the profiles along the propagation axis for the central point in the $x-y$ plane (perpendicular to the propagation axis). The behavior of the $R L G_{0}^{0}$ corresponds to a typical Gaussian fundamental beam. For higher orders, the behavior is similar in all the cases studied: it begins with no intensity at the central point, and then the intensity increases until reach a maximum. The distance at which the maximum is reached is longer when $m$ is higher. This maximum also increases with the mode order $m$. After the maximum is reached, the intensity decreases along the propagation axis following the behavior of a fundamental Gaussian beam.

However, the propagations drawn in Figure 3 show that the rectified beams are not pure Gaussian beam, rather near-likely Gaussian beams. In order to give a numerical description of the quality of the mean, we use a merit function. The Mean Square Error (MSE) for an $x-y$ plane at any distance $z$ of propagation, is defined as 


$$
\operatorname{MSE}(z)=\frac{\int\left|I_{\text {reference }}(x, y ; z)-I(x, y ; z)\right|^{2} d x d y}{\int\left|I_{\text {reference }}(x, y ; z)\right|^{2} d x d y},
$$

where $I_{\text {reference }}(x, y ; z)$ is an intensity pattern used as a reference, which can change along $z$-axis, and $I(x, y ; z)$ is the intensity pattern of the $R L G_{p}^{m}$ beam at a distance $z$. We are interested in two parameters: the closeness to a pure fundamental Gaussian beam, and the closeness to a "flat-top" profile (in other words, the amount of light that is concentrated in the central region with a homogeneous distribution). These two concepts will bring us a description of the validity of $R L G$ beams as illumination sources. For the first point, we use as $I_{\text {reference }}$ the square of a Gaussian distribution, defined as

$$
\psi_{G}=K \exp \left(-\frac{x^{2}}{\omega_{x}^{2}}-\frac{y^{2}}{\omega_{y}^{2}}\right)
$$

where $K$ is a constant that gives the maximum value of the function. The reference function is then calculated as $I_{\text {reference }}=G=\left|\psi_{G}\right|^{2}$. For the second point of interest, we use as $I_{\text {reference }}$ the square of a Super-Gaussian distribution of order $n$, defined as

$$
\psi_{S G}^{n}=K \exp \left(-\frac{x^{n}}{\omega_{x}^{n}}-\frac{y^{n}}{\omega_{y}^{n}}\right)
$$

where $n$ is an integer. A higher value of $n$ produces a distribution closer to a flat-top profile. In our case, we chose $n=90$. Then, the reference intensity for this case results $I_{\text {reference }}=S G=\left|\psi_{S G}^{90}\right|^{2}$.

For the calculus of $M S E$, we calculate at any distance $\omega_{x}$ and $\omega_{y}$. Then, we define a $G$ and a $S G$ functions with these widths, and a maximum value equal to the maximum intensity at this plane. In Figure 6 we compare a $L G_{0}^{1}$ intensity one-dimensional profile with the corresponding $G$ and $S G$ functions with the proper width (for the calculations, we have bi- 
dimensional functions). Next, we apply eq. (6) to these functions using each $I_{\text {reference }}$, and we obtaining a value of $M S E$ at this distance. Iterating these steps along $z$-axis, we can plot the curves shown in Figure 7.

In Figure 7a, the MSE relative to a Gaussian distribution along the axis of propagation is plotted. The smaller $M S E$ observed correspond to the fundamental $R L G_{0}^{0}$ beam. For higher orders of $R L G_{0}^{m}$ beams, a quickly minimization of the error before a distance of $z_{R}$ is noticed. After a distance of $2 z_{R}$ all the curves show a falling dependence with the distance. It is notorious that the minimum MSE with the distance, between the upper orders plotted, is obtained for $m=2$. This is due the lack of rotational symmetry.

The MSE relative to a super-Gaussian distribution is plotted in Figure $7 \mathrm{~b}$. The curve for the fundamental $R L G_{0}^{0}$ beam follows a falling dependence, due again to the limited size of the computational window: as the beam diverges through propagation, there is a high amount of intensity in the internal zone of the super-Gaussian distribution. For $R L G_{0}^{m}$ beams of higher orders, the amount of intensity concentrated in the internal zones of the super-Gaussian profile decreases with the order $m$ (as the error increases with $m$ ), except for a transition zone at a distance lower than $z_{R}$. In this transition zone the $R L G_{0}^{m}$ beams (with $m \neq 1$ ) reach a minimum error lower than the error of the fundamental $R L G_{0}^{0}$ at the same distance. This minimum error is lower when $m$ increases. Thus, in this transition zone the rectified beams trends to a flat-top profile rather than to a Gaussian distribution. This fact can represent even an advantage in order to use $R L G$ beams as illumination sources in micro optical devices. 


\section{Conclusions}

Laguerre-Gaussian beams are produced by laser resonators, especially when high output powers are required. They usually present a singularity in the phase distribution, causing a lack of intensity in central region. As a consequence, they do not result a good choice for illumination in micro-optical applications. The simplest way to correct the singularity is to use binary phase masks producing a homogeneous phase distribution. The behavior of rectified beams produced in such a way has been studied under Fresnel approach. For distances longer than $2 z_{R}$ the rectified beams tend to a Gaussian distribution, but with lower quality than a pure Gaussian beam. For the particular case of $m=2$ we obtain a lower Gaussian width in addition to a closer-Gaussian distribution along the distance of propagation. For distances of propagation smaller than $2 z_{R}$ a transition region is observed. In this region, the rectified beam is changing from the original Laguerre-Gaussian mode to a near-Gaussian beam. In this transition region, the rectified beams are closer to a super-Gaussian distribution rather than a common Gaussian distribution. The results of this work are very valuable for the use of laser sources producing this kind of beams (as in the case of VCSELs) in micro-optical applications. 


\section{References}

[1] K. Iga, F. Koyama, and S. Kinoshita, "Surface emitting semiconductor lasers," IEEE J. Quantum Elect. 24(9), 1845-1855, 1988.

[2] A. Larsson, "Advances in VCSELs for Communication and Sensing," IEEE J. Sel. Top. Quant. 17(6), 1552-1567, 2011.

[3] K-Y. Hung, H-T. Hu, and F-G. Tseng, “Application of 3D glycerol-compensated inclinerexposure technology to an integrated optical pick-up head,” J. Micromech. Microeng. 14, 975-983, 2004.

[4] J. Bristow, J. Lehman, Y. Liu, M. Hibbs-Brenner, L. Galarneuau, R. Morgan, "Recent Progress in Short Distance Optical Interconnects,” Proc. SPIE 3005, 112-119, 1997.

[5] F. Schaal, S. Weidenfeld, FJ Salgado-Remacha, M. Rutloh, J. Stumpe, M. Jetter, R. Rossbach, P. Michler, C. Pruss, and W. Osten, "Micro optical active spatial polarization control,” EOS Topical Meeting on Optical Microsystems, 2011.

[6] C. Degen, I. Fischer, and W. Elsässer, "VCSELs: Influence of pump profile, spatial hole burning, and thermal effects," Opt. Express 5(3), 38-47, 1999.

[7] C.J. Chang-Hasnain, J.P. Harbison, G. Hasnain, A.C. Von Lehmen, L.T. Florez, and N.G. Stoffel, "Dynamic, Polarization, and Transverse Mode Characteristics of Vertical Cavity Surface Emitting Lasers, IEEE J. Quantum Elect. 27(6), 1402-1409, 1991.

[8] S. Weidenfeld, M. Eichfelder, M. Wiesner, WM. Schulz, R. Rossbach, M. Jetter, and P. Michler, “Transverse-Mode Analysis of Red-Emitting Highly Polarized Vertical-Cavity Surface-Emitting Lasers,” IEEE J. Sel. Top. Quant. 17(3), 724-729, 2011.

[9] H. Kogelnik, T. Li, "Laser Beams and Resonators," Appl. Optics 5(19), 1550-1567 (1996). 
[10] F.J. Salgado-Remacha, L.M. Sanchez-Brea, E. Bernabeu, "Micromachining of Diffractive Optical Elements Embedded in Bulk Fused Silica by Nanosecond Pulses,” J. Lightwave Technol. 29(6), 850-855 (2011).

[11] G. Machavariani, N. Davidson, Y. Lumer, I. Moshe, A. Meir, S. Jackel, "New methods of mode conversion and beam brightness enhancement in a high-power laser," Opt. Mater. 30, 1723-1730, (2008).

[12] E. Cagniot, M. Fromager, T. Godin, N. Passilly, and K. Aït-Ameur, "Transverse superrresolution technique involving rectified Lagerre-Gaussian $L G_{p}^{0}$ beams," J. Opt. Soc. Am. A 28(8), 1709-1715, 2011.

[13] Y. Toda, K. Shiegematsu, K. Yamane, R. Morita, "efficient Laguerre-Gaussian mode conversion for orbital momentum resolved spectroscopy," Opt. Commun. 308, 147-151 (2013).

[14] H.C. Eckstein, U.D Zeitner, "Experimental realization of a diffractive unstable resonator with Gaussian amplitude of the outcoupled beam using a VECSEL amplifier,” Opt. Express 17(20), 17384-17390 (2009).

[15] M.P. Buric, J. Falk and S.D. Woodruff, "Conversion of a TEM10 beam into two nearly Gaussian beams,” Appl. Optics 49(4), 739-744 (2010).

[16] N. Davidson, A.A. Friesem and E. Hasman, "Diffractive elements for annular laser beam transformation,” Appl. Phys. Lett. 61(4), 381-383 (1992).

[17] A.T. O’Neil, J. Courtial, "Mode transformations in terms of the constituent HermiteGaussian or Laguerre-Gaussian modes and the variable-phase mode converter," Opt. Commun. 181, 35-45 (2000). 
[18] S. Topuzoski, Lj. Janicievic, "Conversion of high-order Laguerre-Gaussian beams into Bessel beams of increased, reduced or zeroth order by use of a helical axicon," Opt. Commun. 282, 3462-3432 (2009).

[19] A. Syouji, K. Kurihara, A. Otomo and Shingo Saito, "Diffraction-grating-type phase converters for conversion of Hermite-Laguerre-Gaussian mode into Gaussian mode," Appl. Opt. 49(9), 1513-1517 (2010).

[20] V. Peet, “Biaxial crystal as a versatile mode converter,” J. Opt. 12, 095706 (2010).

[21] Y.C. Lin, T.H. Lu, K.F. Huang and Y.F. Chen, "Generation of optical vortex array with transformation of standing-wave Laguerre-Gaussian mode,” Opt. Express 19(11), 10293 $10303(2011)$

[22] A.E. Siegman, "Binary phase plates cannot improve laser beam quality," Opt. Lett. 18(9), 675-677 (1993).

[23] M. D. Feit and J. A. Fleck Jr., "Light propagation in graded-index optical fibers" App. Opt. 17, 3990-3998 (1978).

[24] Z. Mei, D. Zhao, J. Gu and H. Mao, “Approximate analytical expressions of LaguerreGaussian beams passing through a paraxial optical system with an annular aperture," Optik 7, 311-316 (2004).

[25] R.K. Arora and Z. Lu, "Graphical study of Laguerre-Gaussian beam modes," Microwaves, Antennas and Propagation, IEEE Proceedings 141(3), 145-150 (1994)

[26] J. Alda, "Laser and Gaussian Beam Propagation and Transformation," in Encyclopedia of Optical Engineering (Marcel Dekker, 2003), pp 999-1013. 
Salgado-Remacha, "Laguerre-Gaussian beams shaping by binary phase plates as illumination sources in microoptics"

\section{Figure 1}
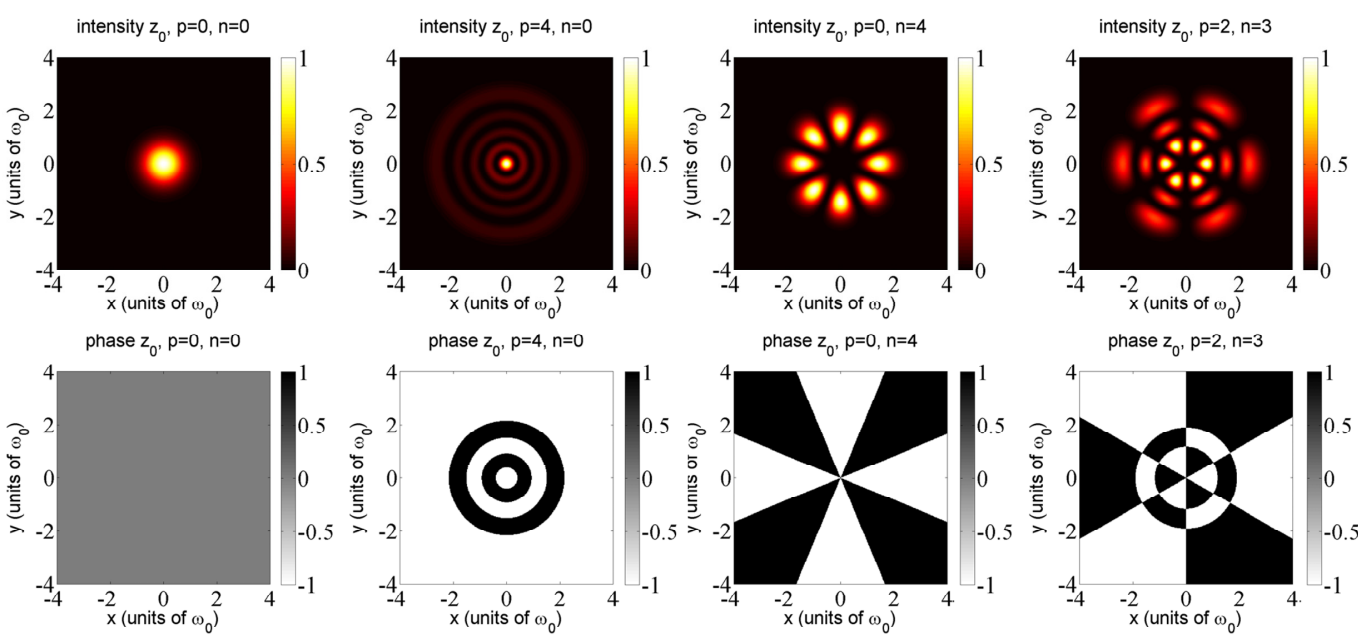
Salgado-Remacha, "Laguerre-Gaussian beams shaping by binary phase plates as illumination sources in microoptics"

Figure 2

(a)

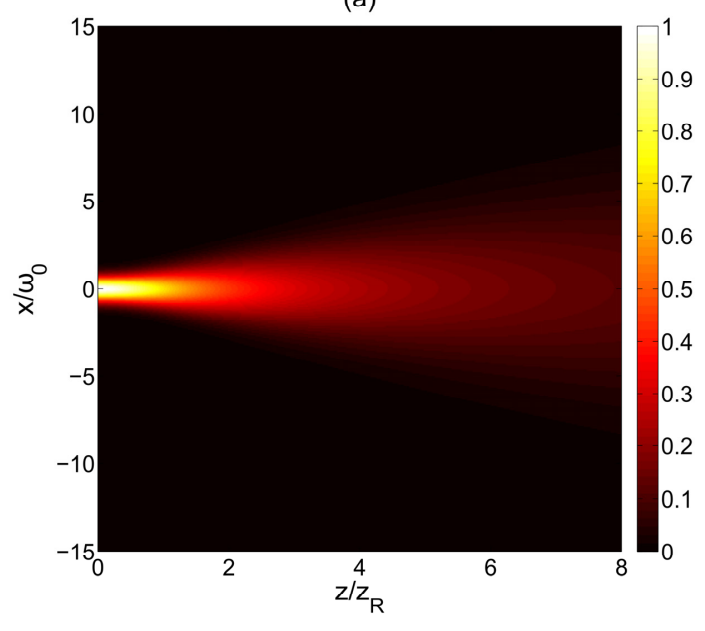

(b)

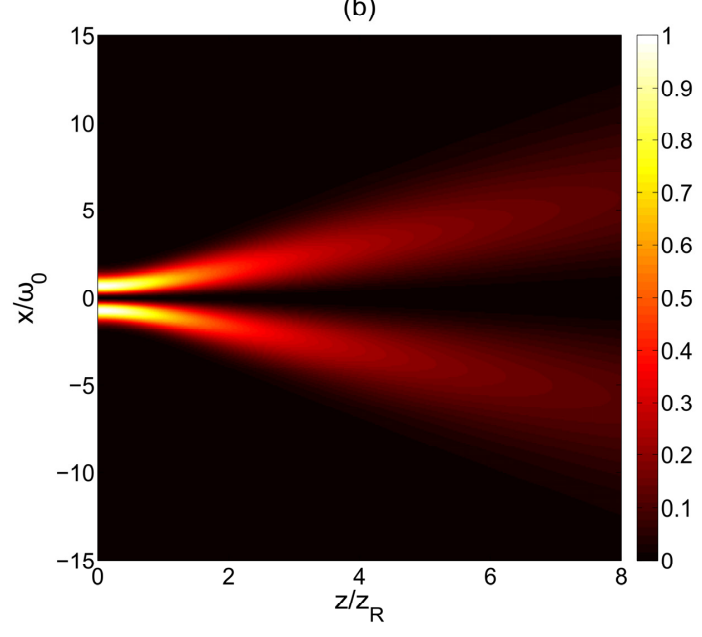



optics"

Figure 3
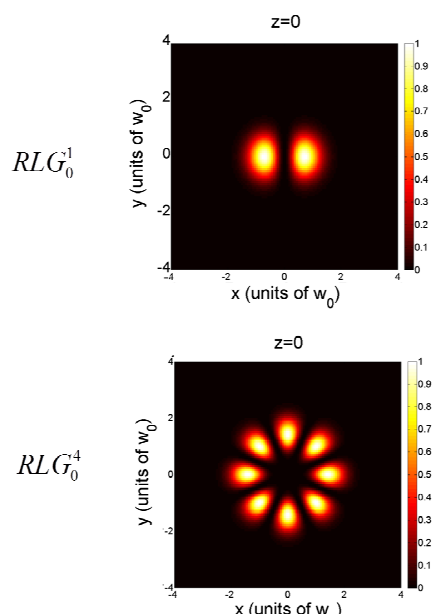

$x\left(\text { units of } w_{0}\right)^{2}$
$\mathrm{z}=\mathrm{z}_{\mathrm{R}}{ }^{2}$

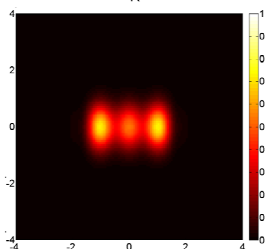

${ }^{2} x\left(\text { units of } \mathrm{w}_{0}\right)^{2}$

$\mathrm{z}=\mathrm{z}_{\mathrm{R}} / 2$

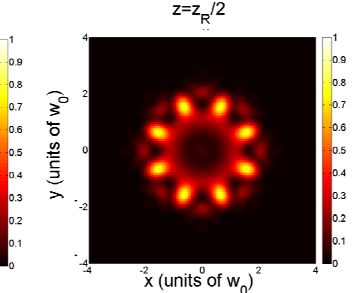

$z=z_{R}$

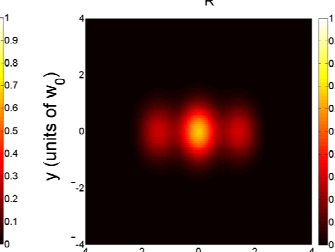

$x^{-2}\left(\right.$ units of $\left.w_{0}\right)$

$z=z_{R}$

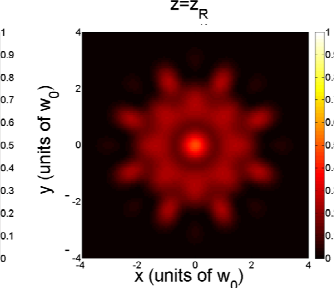

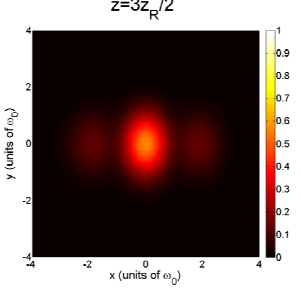

$z=3 z_{R} / 2$

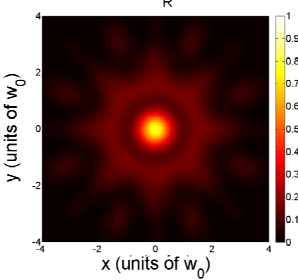

${ }^{2} x$ (units of $\left.w_{0}\right)^{2}$ 
Salgado-Remacha, "Laguerre-Gaussian beams shaping by binary phase plates as illumination sources in microoptics"

Figure 4
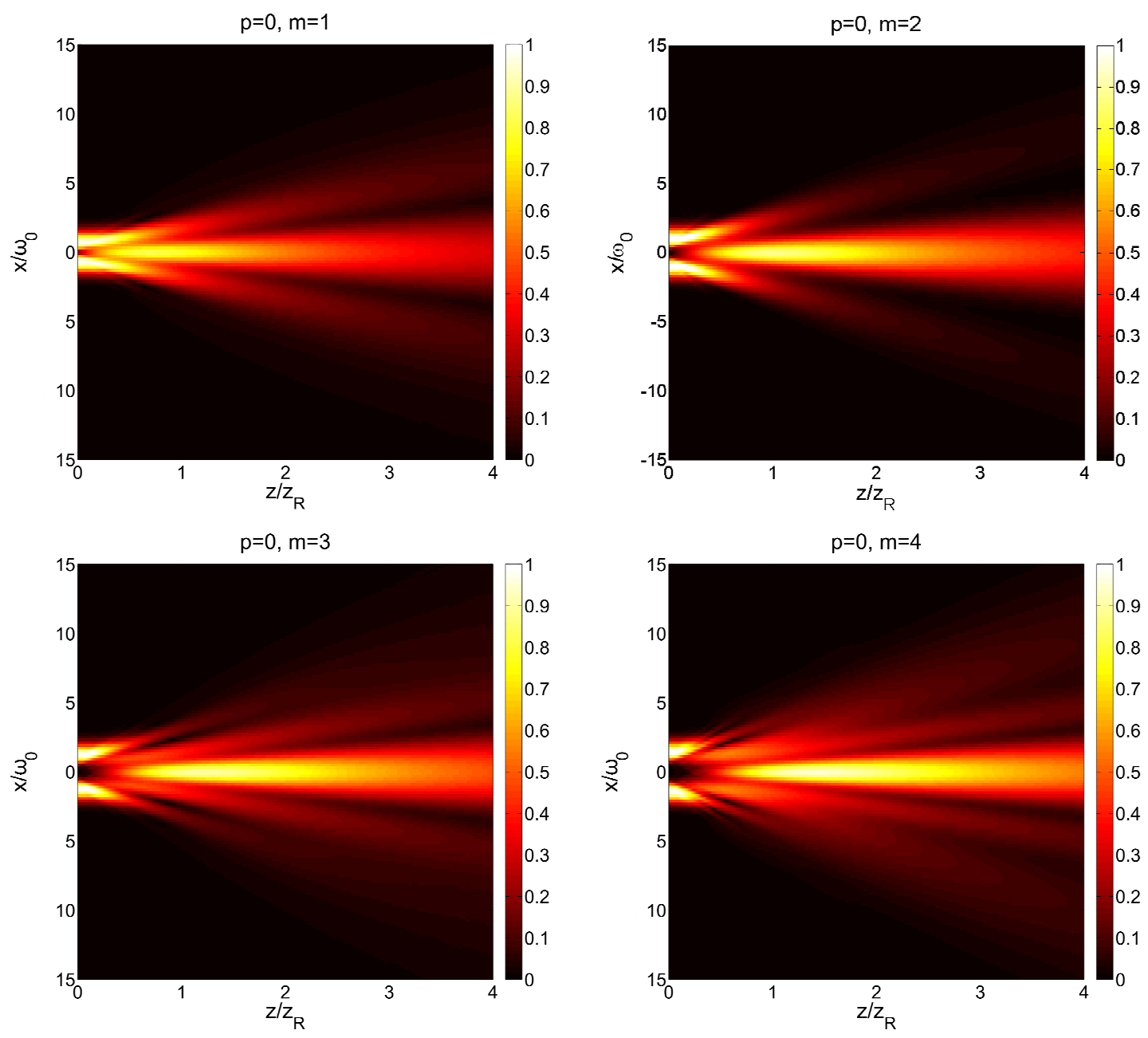
Salgado-Remacha, "Laguerre-Gaussian beams shaping by binary phase plates as illumination sources in microoptics"

\section{Figure 5}
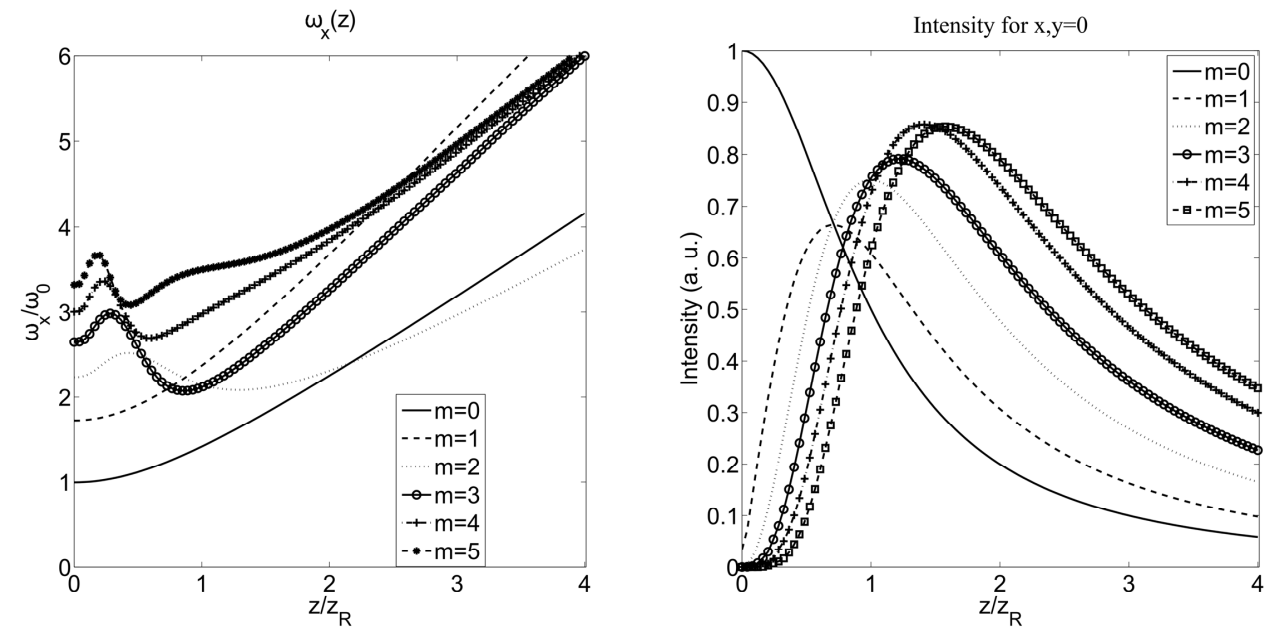
Salgado-Remacha, "Laguerre-Gaussian beams shaping by binary phase plates as illumination sources in microoptics"

\section{Figure 6}

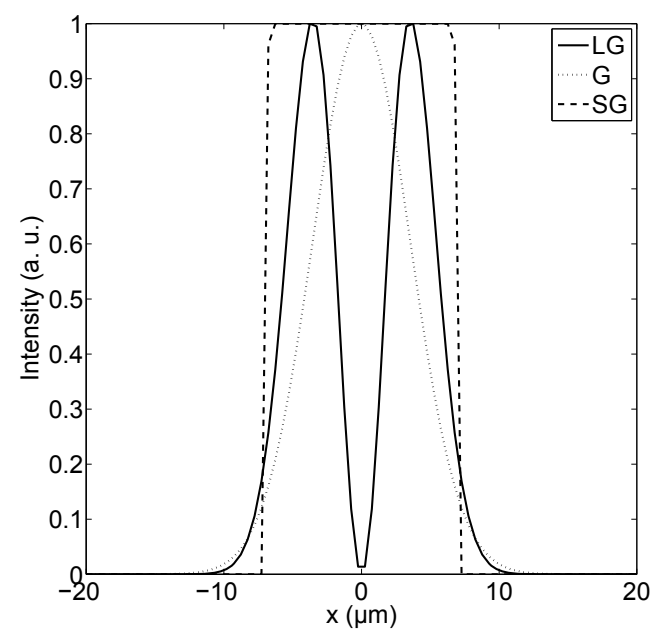


Salgado-Remacha, "Laguerre-Gaussian beams shaping by binary phase plates as illumination sources in microoptics"

\section{Figure 7}

(a)

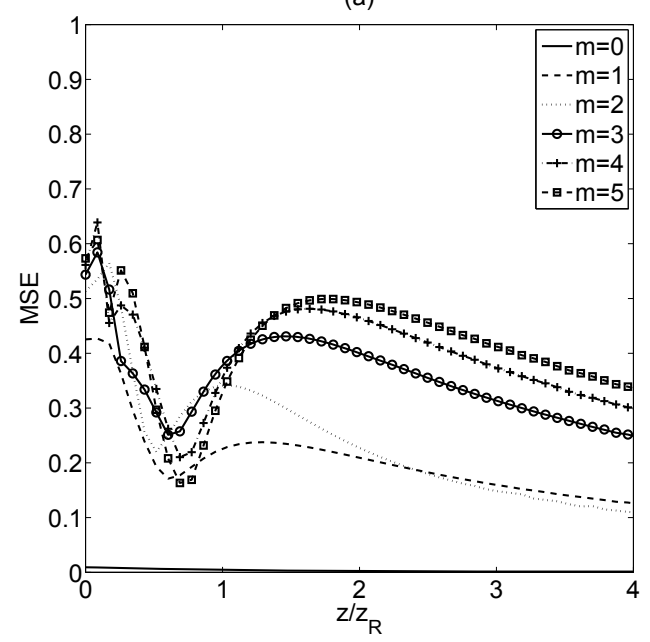

(b)

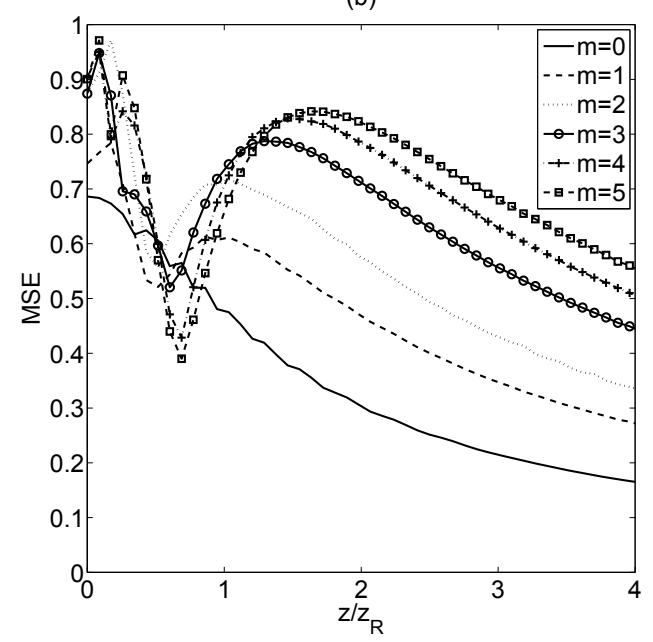


Salgado-Remacha, "Laguerre-Gaussian beams shaping by binary phase plates as illumination sources in micro-

$$
\text { optics" }
$$

\section{Figure captions}

Figure 1. Intensity and phase patterns for $L G_{p}^{m}$ beams at $z=0$ with $\lambda=0.6328 \mathrm{~nm}$, $w_{0}=5 \mu \mathrm{m}$ and different values of $p$ (the radial order ) and $m$ (the azimuthal order).

Figure 2. Simulation by means of BPM: a) propagations of a $L G_{0}^{0}$ mode beam, and b) propagation of a $L G_{0}^{1}$ mode beam. Both propagations correspond to a profile along $x$-axis for $y=0$, and are expressed in terms of $w_{0}$ and $z_{R}$. For the calculations, $\lambda=632.8 \mathrm{~nm}, w_{0}=5 \mu \mathrm{m}$, and $z_{R}=124 \mu \mathrm{m}$.

Figure 3. Propagation of a $R L G_{0}^{1}$ beam (up) and a $R L G_{0}^{4}$ beam (down), at different distances. Simulation obtained by means of BPM with $\lambda=632.8 \mathrm{~nm}$ and $w_{0}=5 \mu \mathrm{m}$.

Figure 4. Simulation by means of BPM of the propagation of several $R L G_{0}^{m}$ with different values of $m$. For the calculations, $\lambda=632.8 \mathrm{~nm}, w_{0}=5 \mu \mathrm{m}$, and $z_{R}=124 \mu \mathrm{m}$.

Figure 5. Left: Gaussian width along $x$-axis obtained using eq. (5), for several $R L G_{0}^{m}$ beams along the propagation axis. Right: Intensity along $z$-axis at the central point for several $R L G_{0}^{m}$ beams. Numerical data have been obtained by means of BPM.

Figure 6. Intensity for different field distribution. (LG): a $L G_{0}^{1}$ function with $\omega_{0}=5 \mu \mathrm{m}$; $(\mathrm{G})$ : a Gaussian function; and (SG): Super-Gaussian function with $n=90$. All of them have a Gaussian width of $\omega_{x}=7.07 \mu \mathrm{m}$ corresponding to $\omega_{x}=\sqrt{1+1} \omega_{x}=\sqrt{2} \omega_{x}$ following eq. (2). 
Salgado-Remacha, "Laguerre-Gaussian beams shaping by binary phase plates as illumination sources in microoptics"

Figure 7. Mean Square Error (normalized to the unity) of a $R L G_{0}^{m}$ beam for different values of $m$, relative to a) a Gaussian beam; and b) a Super-Gaussian beam with $n=90$; both with the same widths $\omega_{x}$ and $\omega_{y}$ as the $R L G_{0}^{m}$ beam at any distance from the origin. 